\title{
Pattern and Outcome Of Infantile Spasms in Sohag University Hospital :A reterospective study
}

\author{
Abdelrahim Abdrabou Sadek1, Mostfa Ashry Mohamed 2, Zeinab Fayez Meki 3. \\ 1 Assistant Professor of Pediatrics ,Faculty of Medicine, Sohag University, Sohag, Egypt. \\ 2 Lecture of Pediatrics ,Faculty of Medicine, Sohag University, Sohag, Egypt.
}

3 Specialist, Pediatric Department ,Faculty of Medicine, Sohag University, Sohag Egypt.

Type of article : Original

\begin{abstract}
Background: Infantile spasms (IS) are a unique form of seizure disorder that their occurrence is almost entirely limited to infancy (the first year of life) and they are refractory to conventional anticonvulsant drugs. IS usually are associated with developmental retardation or deterioration and a characteristic electroencephalographic (EEG) pattern (hypsarrhythmia) that together form a syndrome. IS involves a sudden, generally bilateral and symmetric contractions of muscles of the neck, trunk, and extremities. Patients with IS can be classified into focal IS and diffuse groups.
\end{abstract}

Objective: Our main objective in this study is to evaluate the clinical profile, laboratory data, and outcome of children having infantile spasms in Sohag University Hospital.

Methods: This retrospective study was conducted on 540 patient (mean age $\mathbf{8 . 4} \pm \mathbf{6 . 9}$ months.: ) from December 2015 to November 2016 in neuropediatric outpatient Clinic, Pediatric department at Sohag University Hospital. Detailed clinical data of the patient, Investigation done for the patient including the neuroimaging (MRI or CT), EEG pattern, basic metabolic studies, serum enzymes and Karyotyping were retrospectively collected from files and records .

Results: In this study; ( $66.1 \%)$ of cases were males.(33,9\% ) of cases were females ,mean age of presentation $(8.4 \pm 6.9$ months). Positive consanguinity was found in (7.6\%) of cases. $\mathbf{( 7 9 . 2 \% )}$ of cases presented with flexor spasms, $\mathbf{( 8 . 9 \% )}$ with extensor spasms and $\mathbf{( 1 1 . 9 \% )}$ with mixed spasms. $\mathbf{( 8 \% )}$ of cases were idiopathic, $\mathbf{( 1 3 . 5 \% )}$ of cases were cryptogenic and $\mathbf{( 7 8 . 5 \% )}$ ) of cases were symptomatic, $\mathbf{( 1 5 . 2 \% )}$ ) of cases showed favorable outcome, and $(\mathbf{8 4 . 8 \%})$ cases showed unfavorable outcome, of them $\mathbf{( 1 1 . 5 \% )}$ of cases developed other type of seizures, $\mathbf{( 5 4 . 3 \% )}$ of cases had mental retardation, $\mathbf{( 2 8 . 8 \% )}$ of cases died and $\mathbf{( 5 . 2 \% )}$ of cases had autistic features. In univeriate analysis (symptomatic) etiology $(\mathbf{P}=<\mathbf{0 . 0 0 1}, \mathbf{O R}=$ 4.7) and absence of primary control of infantile spasm $(P=<\mathbf{0 . 0 0 1}, \mathbf{O R}=\mathbf{1 8 . 3})$, and frequency of spasm $(\mathbf{P}=<\mathbf{0 . 0 1 7}, \mathbf{O R}=\mathbf{1 . 8})$ were significantly related to unfavorable outcome. In Multiple variable analysis primary response to treatment (odd ratio $=\mathbf{1 6 . 9}, \mathrm{P}$-value $=<\mathbf{0 . 0 0 1}$ ), etiology (symptomatic) $($ odd ratio $=\mathbf{3 . 2}, \mathrm{P}$-value $=\mathbf{0 . 0 0 2})$ significantly associated with unfavorable outcome .

Conclusion: . Infantile spasms was common. it occured in males more than females with ratio of 1.9:1; the majority of cases were in the age range 1-12 months. flexor spasms was more common than extensor, mixed type of spasms. Symptomatic type was most common than cryptogenic, idiopathic. long-term neurodevelopment outcomes for children with IS had several factors, including symptomatic etiology, abnormal development at the time of diagnosis, poor response to initial treatment. .

Study Limitation: Patient presented less than 2 months or above 2years

Key words: pattern of infantile spasm, outcome of infantile spasm, EEG pattern of infantile spasm 


\section{Introduction:}

Infantile spasm is a catastrophic, agespecific epilepsy syndrome that has its onset within the first 12 month of life (Guzzettaf et al., 2007). This disorder is characterized by epileptic spasms which consist of massive myoclonic jerks of the body, which can be extensor or flexor (or both) in nature. Infantile spasms often are accompanied by developmental regression and a characteristic interictial electroencephalogram (EEG) pattern known as hypsarrhythmia. When all three feature are present, the term "West syndrome" is commonly used (Zidenval, Olofsson, 1995, Cowan, Bodensteiner, 1989). The syndrome is considered to be catastrophic because of the frequent sequelae of global neurodevelopmental delay, significant intellectual disability, and medically refractory epilepsy In most cases, the initial age of onset is between 3 and 7 months of age, and over $90 \%$ of cases begin before 12 months of life. The incidence of infantile spasm is 2 to 3 per 10,000 live births with a lifetime prevalence of 1.5 to 2 per 10,000 children (Sidenval, Olofsson, 1995, Cowan, Bodensteiner, 1989). It is slightly more common in males, accounting for about $60 \%$ of cases, and a family history exist in 3 to 6 of cases (Trevathan et al., 1999, Riikonen., 2001). Prompt diagnosis and urgent treatment of affected children can improve their long- term outcomes. Infantile spasms may be classified into three groups: symptomatic, cryptogenic and idiopathic (ILAE, 1989). The term symptomatic is used to describe cases in which there are structural brain abnormalities or metabolic causes seen in a child with preexisting developmental delay. When there are no apparent causes identified in a child with developmental delay or some other neurologic impairment before the onset of spasms, the term cryptogenic infantile spasms is used and constitute up to $15 \%$. Idiopathic infantile spasms are those in which the child is developmentally normal, with a normal neurologic exam prior to onset of infantile spasm (ILAE, 2001). Clinically, the spasms appear in clusters and are characterized by brief, sudden contractions of the axial musculature. The clusters may occur several times daily, with up to 100 spasms per day. They appear to be temporally related to sleep, tending to occur as the infant falls asleep or awakens (Osborn et al., 2010). Depending on the muscle groups involved, the spasms can be further subdivided into flexor, extensor, or mixed. The type of spasms may also be influenced by the position of the body at the time the spasms occur (Pellock et al., 2010). Diagnosis includes a complete history, physical and neurological examination, and an urgently obtained EEG. The latter is critical because the ultimate diagnosis of infantile spasms is made by the clinical history coupled with the EEG findings which has characteristic inter ictal pattern associated with infantile spasms is hypsarrhythmia, which consists of a disorganized and asynchronous high- amplitude background with spikes and slow waves. This pattern can be seen in wakefulness and is enhanced during sleep. Therefore, an EEG study to rule out infantile spasm should ideally include both awake and sleep recording (Mytinger, Joshi, 2012).

The spontaneous remission rate of infantile spasms in limited natural history studies is $30 \%$. Although clinical spasms and the typical EEG pattern disappear by 3 to 4 years of age, up to $60 \%$ of children with infantile spasms go on to develop other 
types of seizures. The long- term prognosis for infantile spasms in terms of neurodevelopmental outcomes and development of other types of seizures remain dismal, although most studies reported better neurodevelopmental outcomes in idiopathic cases (Go et Material and methods:

This retrospective hospital based study was conducted on all infants and children diagnosed as having infantile spasms in the last five years clinically and by EEG, from December 2015 to November 2016. in neuropediatric outpatient Clinic, Pediatric department at Sohag University Hospital. Detailed clinical data of the patient were retrospectively collected from files and records with special reference to: the presenting symptoms as type of spasms ,frequency, duration, types of medications received, response to treatment, developmental history, sex, age at onset of spasms, family history of epilepsy, significant pre-/peri/postnatal insults, and the follow-up period were recorded. The response to the initial treatment was included in the database. Data of the patient's examination focusing on neurological system as conscious level, abnormal features, other congenital anomalies, motor system and cranial nerves examination,EEG pattern. Investigation done for the patient al., 2012). The 2012 updated guidelines recommended a shorter lag time to treatment of infantile spasms with either hormonal therapy or vigabatrin (VGB) to possibly improve long -term developmental outcomes (Darke et al., 2010).

including the neuroimaging (MRI or CT) and basic metabolic studies ( serum amino acids, organic acids in urine, lactate, pyruvate and ammonia levels), serum enzymes as (Aryle sulphatase , Hexose aminadaseA ,B), Karyotyping_,were also taken. the study was approved by the medical ethics committee of Sohag University of Medical Science.

\section{Statistical analysis:}

Data was analyzed using SPSS computer program version 24.0.Qualitative data was expressed as number and percentage. Chi-square $(\chi 2)$ test and Fisher's Exact Test were used for comparison of qualitative variables as appropriate, univariate and multiple logistic regression tests was used to determine factors associated with unfavorable outcome among the studied patients. A 5\% level was chosen as a level of significance in all statistical tests used in the study. A Pvalue of $<0.05$ were considered as statistically

\section{Results:}

Out of the studied cases; ( $\mathbf{6 6 . 1 \%}$ ) of cases were males. $(\mathbf{3 3 , 9 \%}$ ) of cases were

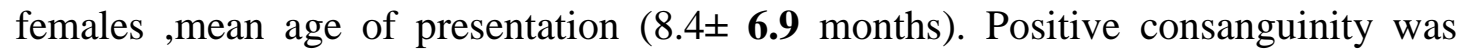
found in $\mathbf{( 7 . 6 \% )}$ of cases. (79.2\%) of cases presented with flexor spasms, (8.9\%) with extensor spasms and $(\mathbf{1 1 . 9 \%})$ with mixed spasms. $\mathbf{( 8 \% )}$ of cases were idiopathic, $\mathbf{( 1 3 . 5 \% )}$ of cases were cryptogenic and $\mathbf{( 7 8 . 5 \% )}$ of cases were symptomatic. Of $\mathbf{4 2 4}$ symptomatic; (61\%) of cases had prenatal disorder, (13.9\%) of cases had perinatal disorder, $\mathbf{( 6 . 6 \% )}$ ) had postnatal disorder, Down syndrome $\mathbf{( 1 . 7 \% )}$ of cases, tuberous sclerosis $\mathbf{( 4 . 4 \% )}$ of cases, metabolic disorder $\mathbf{( 7 . 8 \% )}$ of cases, brain tumor $\mathbf{( \mathbf { 0 . 2 } \% )}$ of case, degenerative disorders $\mathbf{( 2 . 6 \% )}$ of case( Table (1)) . OF the studied cases $(\mathbf{1 5 . 2 \%})$ cases showed favorable outcome, and $\mathbf{( 8 4 . 8 \% )}$ cases showed unfavorable outcome, of them $(\mathbf{1 1 . 5 \%})$ cases developed other type of seizures, $\mathbf{( 5 4 . 3 \%}$ ) cases had mental retardation, $\mathbf{( 2 8 . 8 \% )}$ cases died and $\mathbf{( 5 . 2 \% )}$ cases had autistic features( Table (2)) .. Of the studied cases regarding primary control 
of infantile spasms; $(\mathbf{6 9 . 3 \%})$ of cases had unfavorable outcome, $\mathbf{( 3 0 . 7 \% )}$ of cases had favorable outcome, and those showed no primary control of infantile spasms; $\mathbf{( 9 7 . 6 \% )}$ of cases had unfavorable outcome and $\mathbf{( 2 . 4 \% )}$ of cases had favorable outcome. Of the studied cases those had idiopathic cause were; $(60.5 \%)$ of cases had unfavorable outcome and $(39.5 \%)$ of cases had favorable outcome. Of the cryptogenic cases; $(82.2 \%)$ of cases had unfavorable outcome and $(17.8 \%)$ of cases had favorable outcome. Of symptomatic cases; (87.7\%) of cases had unfavorable outcome and (12.3\%) of cases had favorable outcome. In univeriate analysis (symptomatic) etiology $(\mathbf{P}=<\mathbf{0 . 0 0 1}, \mathbf{O R}=\mathbf{4 . 7})$ and absence of primary control of infantile spasm $(P=$ $<\mathbf{0 . 0 0 1}, \mathrm{OR}=\mathbf{1 8 . 3})$, and frequency of $\operatorname{spasm}(\mathbf{P}=<\mathbf{0 . 0 1 7}, \mathrm{OR}=\mathbf{1 . 8})$ were significantly related to unfavorable outcome. In Multiple variable analysis primary response to treatment (odd ratio $=\mathbf{1 6 . 9}, \mathrm{P}-$ value $=<\mathbf{0 . 0 0 1})$, etiology $($ symptomatic $)$ (odd ratio $=\mathbf{3 . 2}, \mathrm{P}$ value=0.002) significantly associated with unfavorable outcome ( Table (3)) .

Table (1): Etiology of infantile spasms among the studied children

\begin{tabular}{|c|c|c|}
\hline \multicolumn{2}{|l|}{ Etiology } & \multirow{2}{*}{$\frac{\mathrm{N}(\%)}{43(8 \%)}$} \\
\hline Idiopathic & & \\
\hline Cryptogenic & & $73(13.5 \%)$ \\
\hline \multirow[t]{4}{*}{ Symptomatic: } & $\begin{array}{l}\text { Prenatal disorders } \\
\text { Hydrocephalus } \\
\text { Microcephaly } \\
\text { Congenital infections } \\
\text { Brain malformations } \\
\text { Brain atrophy } \\
\text { Joubert syndrome } \\
\text { Chromosomal disorders(Down synderom) } \\
\text { Cerebral calcifications }\end{array}$ & $\begin{array}{l}12(2.2 \%) \\
5(0.9 \%) \\
5(0.9 \%) \\
26(4.8 \%) \\
195(36.1 \%) \\
4(0.7 \%) \\
9(1.7 \%) \\
3(0.6 \%)\end{array}$ \\
\hline & $\begin{array}{l}\text { Perinatal disorder } \\
\text { Hypoxic ischemic encephalopathy } \\
\text { Intracranial hemorrhage } \\
\text { kernicterus } \\
\text { Head trauma }\end{array}$ & $\begin{array}{l}55(10.2 \%) \\
2(0.4 \%) \\
1(0.2 \%) \\
1(0.2 \%)\end{array}$ \\
\hline & $\begin{array}{l}\text { Postnatal disorder } \\
\text { Meningitis } \\
\text { Encephalitis } \\
\text { Cerebral stroke } \\
\text { Phenylketonuria }\end{array}$ & $\begin{array}{l}7(1.3 \%) \\
7(1.3 \%) \\
8(1.5 \%) \\
6(1.1 \%)\end{array}$ \\
\hline & $\begin{array}{l}\text { Other causes } \\
\text { Brain tumor } \\
\text { Metabolic disorders } \\
\text { Neurocutaneous disease (tuberous sclerosis) } \\
\text { Degenerative disorders (White matter disease) }\end{array}$ & $\begin{aligned} 1(0.2 \%) \\
41(7.8 \%) \\
24(4.4 \%) \\
14(2.6 \%)\end{aligned}$ \\
\hline Total & & $540(100 \%)$ \\
\hline
\end{tabular}

Table (2): Outcome of infantile spasms among the studied children.

\begin{tabular}{|l|l|}
\hline Parameters & N (\%) \\
\hline Favorable & $\mathbf{8 2}(15.2 \%)$ \\
Control of convulsions, decrease frequency, normal psychomotor development & \\
\hline Unfavorable & $53(11.5 \%)$ \\
Epileptic & $24(5.2 \%)$ \\
Autistic features & $249(54.3 \%)$ \\
Mental retardation & $132(28.8 \%)$ \\
Death &
\end{tabular}


Table (3): Multiple logistic regression of significant variables in the univariate model

\begin{tabular}{|c|c|c|}
\hline Characteristics & OR (CI 95\%) & $P$ - value \\
\hline $\begin{array}{l}\text { Frequency } \\
\text { Frequent } \\
\text { Infrequent }\end{array}$ & $\begin{array}{l}1.06(0.6-1.9) \\
1\end{array}$ & 0.851 \\
\hline $\begin{array}{l}\text { Primary response to treatment } \\
\text { Uncontrolled } \\
\text { Controlled }\end{array}$ & $\begin{array}{l}16.9(7.5-38.4) \\
1\end{array}$ & $<0.001 *$ \\
\hline $\begin{array}{l}\text { Etiology } \\
\text { Idiopathic } \\
\text { Cryptogenic } \\
\text { Symptomatic }\end{array}$ & $\begin{array}{l}1 \\
1.8(0.7-4.7) \\
3.2(1.5-6.7)\end{array}$ & $\begin{array}{l}0.234 \\
0.002 *\end{array}$ \\
\hline
\end{tabular}

\section{Discussion:}

Infantile spasms, is a triad of clinical flexor or extensor spasms, often involving the extremities and head/neck, hypsarrhythmia on electroencephalogram (EEG), and subsequent or concurrent intellectual disability. Infantile spasms is one of the "catastrophic childhood epilepsies" because of the difficulty in controlling seizures and the association with mental retardation. However, early recognition, a careful diagnostic evaluation, and proper treatment may allow some children to attain seizure control and to achieve a normal, or at least much improved, level of development (Pellock et al .2010).During the study period, 540 cases were included, of whom $(66.1 \%)$ cases were males , $(33.9 \%)$ cases were females, the male to female ratio was 1.9:1. Our study was in agreement with epidemiologic data of west syndrome in Finland which showed male predominance, accounting for about $60 \%$ of cases, male to female ratio 1.5:1 (Riikonen, 2001) .According to the age of diagnosis in this study the majority of cases were in the age range 1-12 month $\mathbf{8 8 . 8 \%}$ followed by the age range above 12 month 11.1\%.The mean age of presentation was $(8.4 \pm 6.9$ months $)$. Our results were near the results of a retrospective study done at Asan medical centre Soul, Korea, to determine long term outcome of infantile spasms, which showed that $95 \%$ of cases begin in the first 12 months of age but the mean age of onset was 5 months, and with the study of Hrachovy, which showed that the spasms mainly begin in the first year mostly between 4 and 8 months of age (Hrachovy, 2002, Seak et al., 2010). And close to the result of Sankar et al, which showed that, IS most commonly develops between 3 and 8 months of age, with only $8 \%$ of cases first being encountered in infants older than 1 year of age. And in agreement with the study of Wang et al, which Showed that infantile spasms occur in first year of life with peak incidence at 6 months of age and less than $10 \%$ of cases presenting after 12 months of age (Sankar et al., 2006, Wang et al., 2013). According to seizures pattern, 79.2\% presented with flexor spasms, 8.9\% presented with extensor spasms, 11.9\% presented with mixed spasms. Our result were indifferent with the study of Jean, which showed that flexor spasms were in $42 \%$ of cases, extensor spasms were the least common, and mixed flexor-extensor spasms accounted for $50 \%$ of cases (Jean, 2009). According to etiologic classification, idiopathic etiology was in $(8 \%)$ cases, cryptogenic in $(13.5 \%)$ cases, and symptomatic in $(78.5 \%)$ cases. Our results were close to the results of the retrospective study done at Asan medical centre, Soul, Korea to 
determine long term outcome of infantile spasms which showed that $30.2 \%$ had cryptogenic and $69.8 \%$ had symptomatic IS. And with the result of Riikonen, in which the cause of spasms was found in more than $70 \%$ of cases (Riikonen, 2001, Seak et al., 2010). According to outcome of infantile spasms, $\quad(84.8 \%)$ cases had unfavorable outcome of which $(54.2 \%)$ cases had mental retardation, $(28.8 \%)$ cases died, $(5.4 \%)$ cases had autistic feature, $(11.8 \%)$ of cases had other types of seizure and $(15.2 \%)$ of cases were normal. Our results were near the results of Riikonen, in which a study was done on 214 cases of which $31 \%$ died, $46 \%$ had mental retardation, and $24 \%$ had favorable outcome (Riikonen, 2001). Because of poor prognosis despite treatment, (Darke et al., 2010 , Pavone et al., 2013 , O'Callagha et al., 2011). In the current study we found that $\mathbf{8 7 . 7 \%}$ of children with symptomatic IS, but only $\mathbf{6 0 \%}$ of those with idiopathic IS, and $\mathbf{8 2 \%}$ of cryptogenic IS had unfavorable outcomes.

Another prognostic factor is primary clinical and electro clinical outcomes. A study in a large population found that $97 \%$ of patients with favorable long-term outcomes were electro clinical responders to initial treatment (Lux, Osborne, 2006 , Riikonen, 2001). Which were consistent with our study in which $97.7 \%$ of the patient who had unfavorable outcome showed no primary response to treatment. We found that non-response to primary treatment was significantly related to neurodevelopmental disorders with high OR in both univariate and multivariate analysis .The prevalence of unfavorable outcomes in nonresponders was one and half-fold higher than in responders. Furthermore, non-responders with symptomatic IS had the highest prevalence of unfavorable outcomes. identification of risk factors predisposing to poor long-term outcomes, including neurodevelopmental disorders and mortalities had been one of main issues. Several factors had been identified, including symptomatic etiology, abnormal development at the time of diagnosis and poor response to initial treatment.

Above all, underlying pathology (symptomatic etiology) is the most important factor in determining patient outcomes. For example, severe brain malformation, post infectious etiology and tuberous sclerosis have been associated with more unfavorable prognosis. In contrast, patients with cryptogenic, idiopathic etiology, in which no causes can be identified, have a more favorable prognosis We found that the remaining risk factors, including developmental delay at time of diagnosis and treatment lag, were not related to neurodevelopmental outcomes. Children with pre-existing developmental delay or neurological deficits at time of diagnosis had been thought to be related to less favorable developmental outcomes (Lux, Osborne, 2004 , Wong, Trevathan, 2001). This findings were in agreement with our study. This tendency may be related to the association between the underlying causes of IS and preexisting developmental disorders. Children with symptomatic IS had a higher prevalence of pre-existing developmental delay (Goh et al., 2005 , Go et al., 2012).

Conclusions: From this study we concluded that infantile spasms was common it occured in males more than females with ratio of $1.9: 1$, the majority of cases were in the age range 1-12 months. Flexor spasms was more common than extensor and mixed type of spasms. Symptomatic type was most common than cryptogenic, 
idiopathic. Several factors determining long-term neurodevelopment outcomes for children with IS. have been identified, including symptomatic etiology, abnormal development at the time of diagnosis, poor response to initial treatment.The majority $(84.4 \%)$ of children with IS had unfavorable neurodevelopment. Children with symptomatic IS had significantly poorer outcomes than those with cryptogenic IS. Non-response to initial electro clinical treatment was significantly related to unfavorable long-term outcomes, indicating that the initial control of seizures may be References:

- Cowan LD, Bodensteiner JB. Prevelance of epilepsies in children and adolescents Epilepsia 1989:30:94 -106.

- Darke K, Edwards SW, Hancock E et al . Developmental and epilepsy Outcome at age 4year in the UKISS trail comparing hormonal treatment to vegabatrin for infantile spasm :a multi -centre randomized trail .Arch Dis Child 2010 :95:382-386.

- Go CY, Mackay MT, Weiss SK, et al. Evidence-based guideline update: medical treatment of infantile spasms. Report of the Guideline Development Subcommittee of the American Academy of Neurology and the Practice Committee of the Child Neurology Society. Neurology 2012;78:1974-80.

- Goh S, Kwiatkowski DJ, Dorer DJ, et al. Infantile spasms and intellectual outcomes in children with tuberous sclerosis complex. Neurology 2005;65:235-8

- Hrachovy R. West's syndrome (infantile spasm). Clinical description and diagnosis. Adv Exp Med Biol 2002;497:33-50.

- ILEA, Commission on classification and terminology of the international league against epilepsy. Proposal for revised classification of epilepsies and epileptic syndromes'. Eiplepsia 1989 :34:389-399. important in reducing the occurrence of poor neurodevelopment.

\section{Acknowledgment:}

Authors thank colleagues in the Pediatric Department, Faculty of Medicine, Sohag University (Egypt) for their assistance, and the parents and children who participated in this study.

\section{Conflict of Interest:}

There is no conflict of interest to be declared.

\section{Authors contributions:}

All authors contributed to this project and article equally. All authors read and approved the final manuscript.

- Jean Aicardi., editor. Disease of Nervous system in childhood. 3th ed. Mac Keith pres; 2009. Infantile Spasms; pp. 593-7.

- Lux AL, Osborne JP. A proposal for case definitions and outcome measures in studies of infantile spasms and West syndrome: consensus statement of the West Delphi group. Epilepsia 2004;45:1416-28.

- Mytinger JR, Joshi S; Pediatric Epilepsy Research Consortium, et al. The current evaluation and treatment of infantile spasms among members of the Child Neurology Society. J Child Neurol 2012;27:1289-94.

- O'Callaghan FJ, Lux AL, Darke K, et al. The effect of lead time to treatment and of age of onset on developmental outcome at 4 years in infantile spasms: evidence from the United Kingdom Infantile Spasms Study. Epilepsia 2011;52:1359-64

- Osborn JP, Lux AL ,Edward SW, et al .The underling etiology of infantile spasm (West syndrome): Information from the United Kingdom Infantile Spasms Study (UKISS) on contemporary causes and their classification . Epilepsia 2010:51(10): 2168-2174

- Pavone P, Striano P, Falsaperla R, et al. Infantile spasms syndrome, West syndrome and related phenotypes: what 
we know in 2013. Brain Dev 2014;36:739-51.

- Pellock JM, Hrachovy R, Shinnar S, et al. Infantile spasms: a U.S. consensus report. Epilepsia 2010;51:2175-89.

- Riikonen R. Epidemiological data of West syndrome in Finland. Brain Dev 2001:23:53.54०

- Sankar R, Koh S, Wu J, Menkes JH. Paroxysmal disorders. In: Menkes JH, Sarnat HB, Maria BL , editors. Child neurology. 7th ed. Philadelphia: Lippincott Williams \& Wilkins; 2006. pp. 857-942.
- Trevathan E, Murphy CC, YearginAllsopp M. The descriptive epidemiology of infantile spasms among Atlana children. Epilepsia 1999 $: 40: 748-751$.

- Wong M, Trevathan E. Infantile spasms. Pediatr Neurol2001;24:89-98.

- Sidenval R, Eeg Olofsson O. Epidemiology of infantile spasms in Sweden .Epilepsia 1995: 36:572-574. 